\title{
A Novel Epigenetic Mechanism in Drosophila Somatic Cells Mediated by PIWI and piRNAs
}

\author{
H. LIN AND H. YIN* \\ Yale Stem Cell Center and Department of Cell Biology, Yale University School of Medicine, \\ New Haven, Connecticut 06509
}

\begin{abstract}
Small noncoding RNAs have emerged as key players in epigenetic regulation. Recently, a novel class of small RNAs that interact with Piwi proteins has been discovered in the mammalian and Drosophila germ line. These Piwi-interacting RNAs (piRNAs) represent a distinct small RNA pathway that is widely thought to function only in the germ line. In this chapter, we review our recent work with our collaborators on the epigenetic function of the Drosophila Piwi protein and its associated piRNAs in somatic cells. This work has revealed a novel epigenetic mechanism mediated by Piwi and its associated piRNAs in somatic cells that might also be applicable to the germ line. On the basis of these results, we propose a "Piwi-piRNA guidance hypothesis" for Piwi/piRNA-mediated epigenetic programming, in which the Piwi-piRNA complex serves as sequencerecognition machinery that recruits epigenetic effectors such as heterochromatin protein 1a (HP1a) to specific sites in the genome to execute epigenetic regulation.
\end{abstract}

It is an emerging theme that stem cell fate and other fundamental properties of development are not determined by turning on or off just a few genes. Instead, they are determined by modulating the transcriptional activity of the genome through changes in the local and global organization of chromatin in a heritable manner, a process called epigenetic programming (Surani 2001). Epigenetics refers to the regulation of gene expression by heritable but potentially reversible changes in chromatin structure and/or DNA methylation, but not in DNA sequences themselves. Regulators of epigenetic programming are called epigenetic factors. They include specific chromatin factors, histone modification proteins, and DNA methylation/demethylation enzymes.

In recent years, a role for noncoding small RNAs in the formation of epigenetic chromatin domains has been uncovered, owing mostly to exciting discoveries in the fission yeast Schizosaccharomyces pombe (Verdel et al. 2004). Endogeneous small interfering RNAs (siRNAs) in the nucleus have been proposed to provide a sequencespecific interface between a DNA sequence and its epigenetic state, presumably by their base pairing with genomic DNA or nascent RNA (Wassenegger 2005). Recent studies in fission yeast suggest that RNAi-mediated heterochromatin assembly occurs via a self-enforcing loop mechanism (Motamedi et al. 2004; Noma et al. 2004; Verdel et al. 2004; Sugiyama et al. 2005). A central player in this feed-forward loop is the RNAi-induced initiation of transcriptional gene silencing (RITS) complex, which contains a chromodomain-containing protein (Chp1), Argonaute 1 (Ago1), a novel protein Tas3, and siRNAs (Motamedi et al. 2004; Noma et al. 2004; Verdel et al. 2004; Sugiyama et al. 2005). Ago1 confers sequence specificity by binding to siRNAs and recruits other chro-

\footnotetext{
* Present address: The Ottawa Health Research Institute, Ottawa, Canada.
}

matin proteins to initiate heterochromatization (Noma et al. 2004). Despite these exciting findings, the role of noncoding small RNAs in epigenetic regulation in higher organisms remains largely unexplored. In this chapter, we present a novel epigenetic mechanism in Drosophila somatic cells that is mediated by the Piwi protein and piRNAs.

\section{ARGONAUTE/PIWI PROTEINS AND PIRNAs}

The piwi/argonaute (ago) gene family was first discovered for their evolutionarily conserved function for stem cell self-renewal (Cox et al. 1998). This gene family can be divided into ago and piwi subfamilies, herein referred to as ago and piwi genes, respectively. Both subfamilies encode highly basic proteins $(\mathrm{pI} \sim 10)$ composed of four domains: the amino-terminal domain of variable length, the central PAZ (Piwi, Argonaute, and Zwille) domain that binds to a $3^{\prime}$ portion of single-stranded small RNAs without sequence specificity, the Mid domain that participates in small RNA binding, and the RNase H-like carboxy-terminal Piwi domain (Wang et al. 2008). The Piwi domain of some Ago proteins contains RNA slicing activity that is responsible for mRNA degradation mediated by siRNAs (Liu et al. 2004). However, such activity is not detected in all Ago or Piwi proteins (Liu et al. 2004), indicating that different Ago and Piwi proteins are biochemically distinct.

Piwi proteins are known to have diverse germ-line functions. For example, two Piwi proteins in Drosophila, Piwi and Aubergine, are initially required as maternal factors for germ-line establishment in early embryos (Harris and Macdonald 2001; Megosh et al. 2006). The dose of maternal Piwi determines the number of primordial germ cells (PGCs) in early embryos. Subsequently, Piwi is required for maintaining the transcriptional quiescence of PGCs (Cox 1999) and for germ-line stem cell self-renewal (Cox 
et al. 1998). In mice, Piwi proteins Mili and Miwi are differentially expressed during spermatogenesis. Mili is expressed in spermatogonia (including male germ-line stem cells) spermatocytes and round spermatids, whereas Miwi is expressed from pachytene spermatocytes to elongating spermatids (Deng and Lin 2002; KuramochiMiyagawa et al. 2004; Unhavaithaya et al. 2009). Correspondingly, mili(-/-) mice are blocked in germ-line stem cell self-renewal, with a few escaping spermatogenic cells showing terminal arrest at the pachytene stage (Deng and Lin 2002; Kuramochi-Miyagawa et al. 2004; Unhavaithaya et al. 2009). This implicates a role for Mili in germline stem cell self-renewal and meiosis. In contrast, miwi(-/-) mice display uniform arrest at the beginning of the round spermatid stage, indicating its role as a key regulator of spermatid differentiation (Deng and Lin 2002; Kuramochi-Miyagawa et al. 2004; Unhavaithaya et al. 2009). A human piwi gene called hiwi is expressed in the testis, with its overexpression correlated to seminomascommon testicular cancers originating from malignant PGCs and/or germ-line stem cells (Qiao et al. 2002). In addition, piwi genes in both mammalian systems and in Drosophila have recently been implicated in transposon silencing in the germ line (Girard and Hannon 2008). Despite the well-recognized functions of Piwi proteins in the germ line, their underlying molecular mechanism remains elusive.

Although it is commonly believed that Piwi proteins only function in the germ line, the molecular mechanisms mediated by these proteins are actually better characterized in somatic cells, especially in somatic cells in Drosophila. Studies in Drosophila have provided several lines of evidence for a role of Piwi in epigenetic regulation in somatic cells. First, Piwi is a nuclear protein in both somatic and germ-line cells, except in early-cleavage-stage (i.e., syncytial) embryos (Cox et al. 2000; Megosh et al. 2006). Second, Piwi is a typical suppressor of position effect variegation in somatic cells (Pal-Bhadra et al. 2004), similar to other key epigenetic factors such as HP1 and HP2. Third, piwi deficiency results in loss of methylation of histone 3 at lysine 9 (H3K9me) and the delocalization of HP1 and HP2 from polytene chromosomes in the soma (Pal-Bhadra et al. 2004). Fourth, Piwi directly interacts with HP1, as described below. Thus, Piwi proteins, when in the nucleus, may behave as a key component of a complex required for heterochromatin assembly, just like Ago1 in fission yeast (Verdel et al. 2004). Interestingly, these studies highlight an ignored fact: Piwi proteins also have important functions in somatic cells, at least in Drosophila. The expression of human Piwi (hiwi) in human CD34 ${ }^{+}$hematopoietic stem and progenitor cells (Sharma et al. 2001) and gastric epithelial cells (Liu et al. 2006) as well as the expression and function of Piwi proteins in neoblasts of planarians (Reddien et al. 2005; Rossi et al. 2006; Palakodeti et al. 2008) indicate that Piwi proteins have somatic functions in other organisms as well. The study of Piwi function in somatic cells should also shed light on their function in the germ line.

An important clue to the molecular function of Piwi proteins lies in their small RNA partners, the recently dis- covered piRNAs (Aravin et al. 2006; Girard et al. 2006; Grivna et al. 2006; Lau et al. 2006; Watanabe et al. 2006). piRNAs differ from siRNAs or microRNAs (miRNAs) in several ways. First, piRNAs interact with Piwi proteins but not argonautes. Mouse Piwi (Miwi) is required for piRNA biogenesis and/or stability (Grivna et al. 2006), whereas mouse Ago2 is required for the siRNA pathway (Liu et al. 2004). Second, piRNAs are mostly 24-31 nucleotides instead of approximately 21 nucleotides. Third, there are more than 50,000 cloned species of piRNAs, in contrast to several hundred species of miRNAs. Fourth, most piRNAs match to the genome in clusters of $20-90 \mathrm{~kb}$ in a strand-specific manner, with each cluster likely representing a long single-stranded RNA precursor or, more often, two nonoverlapping and divergently transcribed precursors (Kim 2006). In contrast, siRNAs and miRNAs are derived from doublestranded and short hairpin RNA precursors, respectively. Finally, some piRNAs may be involved in epigenetic regulation (see below). whereas siRNAs and miRNAs generally target mRNAs.

In Drosophila, piRNAs were first isolated as repeatassociated small interfering RNAs (rasiRNAs) (Saito et al. 2006; Vagin et al. 2006). Unlike siRNAs and miRNAs, these rasiRNAs bind to Piwi or Aubergine but not to Ago proteins (Vagin et al. 2006). Moreover, the production of these rasiRNAs requires neither Dicer-1 nor Dicer-2, which generate miRNAs and siRNAs, respectively. Thus, by definition, they are piRNAs. Despite this, the vast majority of piRNAs differ from previously identified rasiRNAs in that they show DNA strand specificity.

\section{A PIWI-PIRNA COMPLEX BINDS TO A PIRNA CORRESPONDING SITE ON CHROMATIN IN SOMATIC CELLS}

To explore the role of Piwi proteins and piRNAs in epigenetic regulation in somatic cells, we focused on Piwi and its interacting piRNAs in Drosophila. We cloned approximately 13,000 Piwi-associated piRNAs from Drosophila (Yin and Lin 2007). The size of these piRNAs shows a Gaussian distribution, with an average length of 25 nucleotides, shorter than mammalian piRNAs but longer than siRNA and miRNAs. Despite this large number, $82 \%$ of these piRNAs were sequenced only once, suggesting that this analysis is far from saturation and that our approximately 13,000 piRNAs are probably only a fraction of the entire pool. Consistent with this, our collection of Piwi-associated piRNAs overlaps with the collection in Gregory Hannon's lab (Brennecke et al. 2007) of 13,904 Piwi-associated piRNAs by only $10 \%$. In addition, including the collection in Haru Siomi's lab of 330 Piwi-associated piRNAs (Saito et al. 2006), only two piRNAs are found in all three collections. Based on these data, we estimate that there are probably as many as 205,000 piRNAs in Drosophila that are associated with the Piwi protein alone.

Piwi-associated piRNAs correspond to all types of genomic sequences (Yin and Lin 2007). One third of 
them correspond to unique sequences in the genome, including gene-coding sequences and intergenic sequences. The remaining two thirds are transcribed from repetitive sequences. In fact, most of the repetitive piRNAs are derived from transposon sequences that account for only about $10 \%$ of the fully assembled euchromatic genome. These piRNAs are especially overrepresented in retrotransposon encoding regions. Large numbers of Piwi-associated piRNAs are mapped into pericentromeric regions, subtelomeric regions, and telomeres. This broad distribution of piRNAs in nongene coding regions implicates their potential role in epigenetic regulation.

To explore the epigenetic function of piRNAs in the soma, we focused on a piRNA that uniquely corresponds to a repetitive sequence in the subtelomeric region (known as the telomere-associated sequence [TAS]) of the right arm of chromosome 3 (3R-TAS), a well-characterized heterochromatic region in Drosophila. This piRNA (called 3R-TAS1 piRNA) is expressed in the ovary and extra-ovarian somatic cells and is at least enriched, if not exclusively present, in the nucleus (Figs. $1 \mathrm{~B}$ and $3 \mathrm{~B}$ [below]). Keeping in mind that Piwi is also a nuclear protein in both somatic and germ-line cells (Cox et al. 2000), we postulated that the Piwi-TAS piRNA complex is associated with the piRNA-corresponding DNA sequence in the 3R-TAS genomic sequence in somatic and possibly germ-line cells. To test this possibility, we conducted chromatin immunoprecipitation (ChIP) experiments to precipitate Piwi from whole flies and quantified the amount of a 73-nucleotide region spanning the 3R-TAS1 piRNA-coding sequence $(\mathrm{D}$, distal in Fig. 1A) that is coprecipitated with Piwi. Piwi is associated with TAS repeats 400 -fold over the nonspecific control and 46-fold over the housekeeping gene $R p 49$. This specific association is further confirmed by ChIP in files carrying a functional myc-piwi transgene using an antiMyc antibody. Myc-Piwi is enriched in 3R-TAS(D) 16.6fold over an intergenic region on chromosome 2 and 11.4and 11.5-fold over succinate dehydrogenase $B$ and actin $88 F$ genes, respectively. In contrast, Piwi is not associated with any of the five piRNA-poor genomic regions examined or even with a proximal 3R-TAS sequence [TAS(P)] only 387 bp away (Fig. 1A). Thus, Piwi is strongly associated with the 3R-TAS(D) that encodes 3RTAS1 piRNA.

We then confirmed Piwi binding to this genomic sequence in somatic cells by immumno-staining for Piwi protein in the polytene chromosomes from larval salivary glands. Piwi is indeed localized onto the chromosomes in many bands enriched in $\mathrm{H} 3 \mathrm{~K} 9 \mathrm{me}$, including the chromosome 3R-TAS region (Fig. 2).

Although it is technically challenging to directly visualize 3R-TAS1 piRNA binding to the 3R-TAS(D) sequence, this binding in complex with Piwi is strongly supported by six lines of evidence: First, this piRNA is at least enriched, if not specifically present, in the nucleus, just like Piwi; second, it binds to Piwi as shown both by coimmunoprecipitation and electrophoretic mobility shift (Yin and Lin 2007); third, only this piRNA corresponds to 3R-TAS(D) and is in fact transcribed from this sequence (Yin and Lin 2007); fourth, Piwi binds specifically to the 3R-TAS(D) site; fifth, Piwi binding to many sites in the polytene chromosome, including the 3R-TAS(D) site, is RNase sensitive (Brower-Toland et al. 2007); finally, the overexpression of 3R-TAS1 piRNA can largely rescue the epigenetic defects of the 3R-TAS(D) sequence in piwi mutants (see below). These data collectively implicate that Piwi and 3R-TAS1-piRNA form a complex that binds to the piRNA-matching DNA sequence.
A

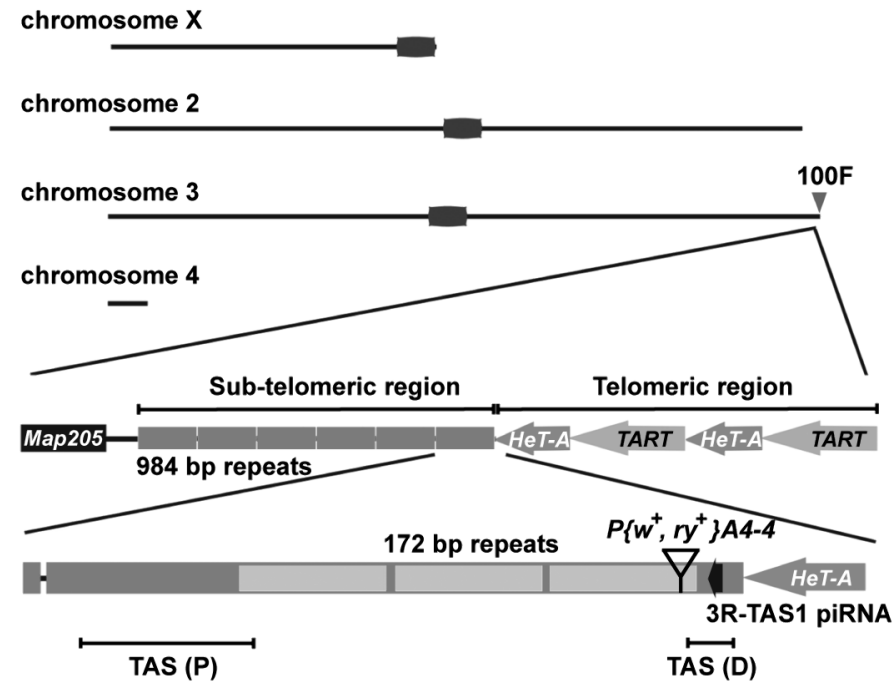

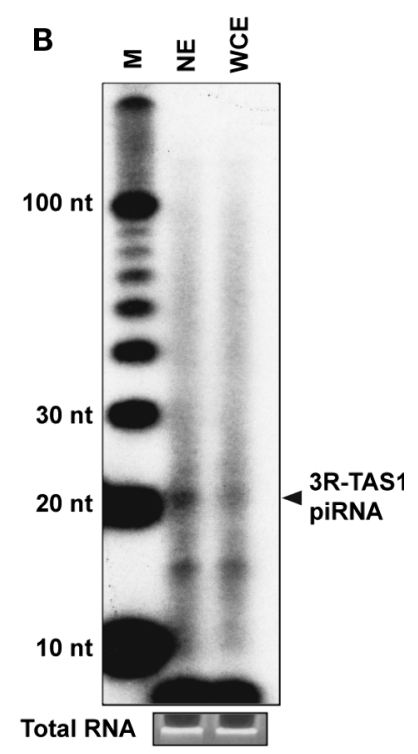

Figure 1. Expression of 3R-TAS1 piRNA that uniquely corresponds to the 3R-TAS(D) sequence. (A) Organization of 3R-TAS. (Black arrow) Position and direction of the unique mapped 3R-TAS1 piRNA. (B) Ribonuclease protection assay showing that 3R-TAS1 piRNA can protect a 597-bp antisense probe that spans the piRNA-coding region and covers 386-982 nucleotides of the 984-bp repeat unit in the antisense direction. The 3R-TAS1 piRNA is at least enriched, if not exclusively present, in nuclear extracts (NE) over wholecell extracts (WCE). (M) 10-bp DNA marker. (Modified from Yin and Lin 2007 [@ Nature Publishing Group].) 


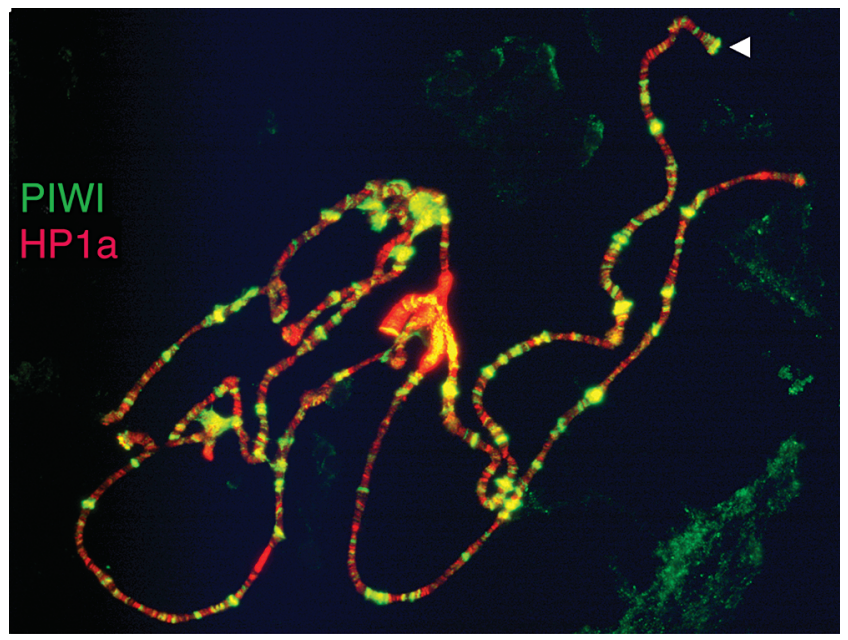

Figure 2. PIWI is associated with chromatin, where it colocalizes with HP1a in many regions. A Drosophila genome is represented by polytene chromosomes from a wild-type Drosophila third-instar larval salivary gland cell. PIWI (green) shows colocalization with HP1a (red) at multiple euchromatic bands along polytene chromosome arms, at telomeres, and in distinct regions of the chromocenter, including the 3R-TAS region (white arrowhead). HP1a is most concentrated in the chromocenter and along chromosome 4. (Adapted from BrowerToland et al. 2007.)

\section{PIWI-PIRNA COMPLEX REGULATES THE EPIGENETIC STATE OF THE TARGET GENOMIC SITES IN SOMATIC CELLS}

To determine whether the binding of Piwi to the TAS region has any effect on its chromatin status, we examined the histone modifications of the 73-bp 3R-TAS(D) sequence in wild-type versus piwi mutant flies by ChIP using antibodies specific against euchromatic or heterochromatic histone modifications. To our surprise, in wild-type flies, transcription activation markers, such as acetylated histone $3 \mathrm{~K} 9$ and dimethylated and trimethylated histone $3 \mathrm{~K} 4$, are abundantly associated with the TAS region, whereas heterochromatin markers, such as HP1a and methylated H3K9, are not as abundantly present as we expected (Fig. 3A). This indicates that this heterochromatin might be transcriptionally active. In piwi mutants, these euchromatic histone modifications are dramatically reduced from the TAS region. On the other hand, heterochromatic markers, such as HP1a and dimethylated and trimethylated histone $3 \mathrm{~K} 9$, are dramatically accumulated on TAS genomic DNA in piwi mutants. This suggests that Piwi, possibly together with its associated 3R-TAS1 piRNA, promotes the euchromatic character of 3R-TAS(D).

To test whether the level of 3R-TAS1 piRNA has an effect on the epigenetic state of 3R-TAS(D), we isolated a P-element mutation $P\left\{w^{+}, r y^{+}\right\} A 4-4$ that is inserted in the 3R-TAS(D) sequence. This insertion causes the specific overexpression of 3R-TAS1 piRNA in wild-type flies (Fig. 3B) and rescues the expression of this piRNA in piwi mutants (data not shown). As a result, this rescue of piRNA expression restores the chromatin state of the 3RTAS(D) sequence, with the euchromatic marker H3AcK4 increased to the wild-type level; heterochromatic markers also reduced to the wild-type level (Fig. 3A). This suggests the involvement of 3R-TAS1 piRNA in the Piwi-mediated epigenetic activation of 3R-TAS(D) sequence.

To determine whether the effect of Piwi on these chromatin modifications indeed has a functional consequence regarding the transcriptional activity of 3R-TAS(D) in somatic cells, we performed two experiments to assay the transcriptional activity of the sequence in somatic cells. First, we used an RNase protection assay to detect the transcription of TAS piRNA itself. 3R-TAS1 piRNA is indeed transcribed from this region, both in the ovary and, importantly, in extra-ovarian somatic cells (Fig. 3B). Second, we further inserted an eye color reporter gene into the 3RTAS(D) sequence, only 128 bp downstream from the TAS piRNA coding sequence. In $p i w i^{+} / p i w i^{+}$flies, the eyecolor reporter is moderately expressed in the eye. In $\mathrm{piwi}^{+} / \mathrm{piwi}^{+}$flies, the expression is significantly reduced, and in piwi-/piwi mutants, the expression is barely detectable (Fig. 3C). Three independent quantifications of the eye pigment indicate that there is at least a fivefold decrease in reporter gene expression from heterozygous to homozygous mutants. These analyses further support the hypothesis that Piwi, presumably together with 3R-TAS1 piRNA, promotes the transcriptional activity of the 3RTAS(D) sequence function in somatic cells.

The effect of Piwi on the transcriptional activity of 3RTAS(D) in somatic cells is clearly inherited during cell division, because both the position effect of the piwi mutant and the transcriptional activation role of Piwi toward 3R-TAS(D) are variably expressed in a polyclonal fashion in fly eyes (Yin and Lin 2007). This indicates that Piwi-mediated transcriptional activation is an epigenetic phenomenon. To our knowledge, this is the first case illustrating that a small RNA-related mechanism has an epigenetic activation function.

\section{PIWI DIRECTLY INTERACTS WITH HETEROCHROMATIN PROTEIN 1A}

To further investigate the Piwi-mediated epigenetic mechanism in somatic cells, we sought to identify direct Piwi interactors and to determine their chromosomal localization. Yeast two-hybrid screens were conducted using full-length (FL), amino-terminal (NT) half, and carboxy-terminal (CT) half Piwi as baits (Fig. 4A) and recovered 102,100 , and 0 strong positives for these baits, respectively (Brower-Toland et al. 2007). Fourty four of 102 positives for Piwi-FL and 39 of 100 positives for 

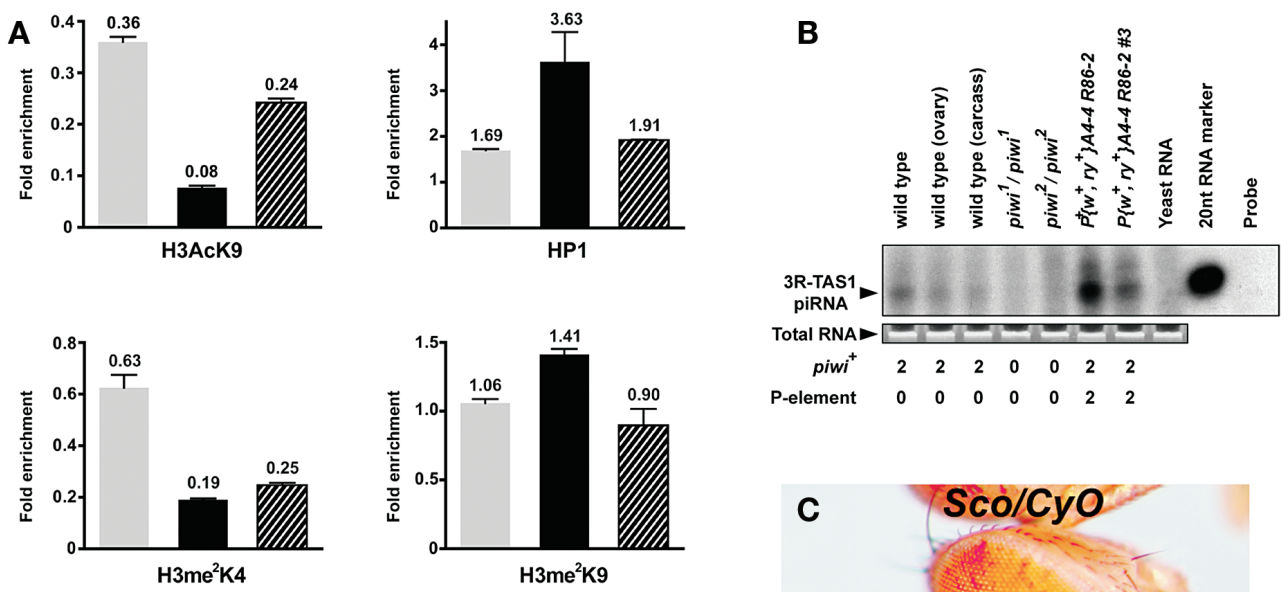

P-element $\quad 0 \quad 0 \quad 0000012$
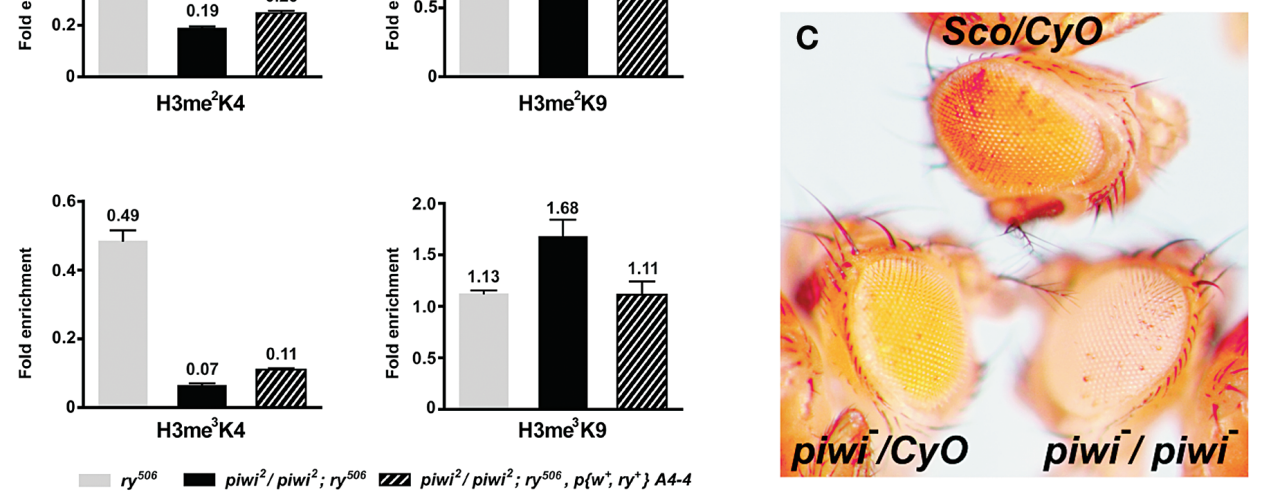

Figure 3. Piwi promotes the euchromatic features and transcriptional activity of 3R-TAS heterochromatin. $(A)$ The association of modified histones $\mathrm{H} 3 \mathrm{Me}^{2} \mathrm{~K} 4, \mathrm{H} 3 \mathrm{Me}^{3} \mathrm{~K} 4, \mathrm{H} 3 \mathrm{AcK} 9, \mathrm{H} 3 \mathrm{Me}^{2} \mathrm{~K} 9, \mathrm{H} 3 \mathrm{Me}^{3} \mathrm{~K}$, and $\mathrm{HP} 1 \mathrm{a}$ with $3 \mathrm{R}-\mathrm{TAS}(\mathrm{D})$ was assayed by ChIP and quantitative polymerase chain reaction (qPCR). The relative enrichment of modified histones is calculated by normalizing the quantity of 3R-TAS(D) DNA against the quantity of Actin5C. Each individual experiment was repeated at least three times. Standard deviations were used to indicate the error bars. $(B)$ Ribonuclease protection assay showing that 3R-TAS1 piRNA is expressed in the wild-type adult fly, both in ovarian and extra-ovarian cells. Its expression is dramatically reduced in piwi ${ }^{l}$ and $p i w i^{2}$ mutants and drastically increased by the $P\left\{w^{+}, r y^{+}\right\} A 4-4$ insertion (four separately maintained lines, 221, 516, R86-2, and $R 86-2 \# 3$ were checked, with two lines shown in the figure). All lanes are loaded with equal amounts of total RNA. The copy numbers of piwi and $P\left\{w^{+}, r y^{+}\right\} A 4-4$ are noted at the bottom of $B$. (C) Piwi promotes the transcription of the eye-color reporter gene white, inserted into the 3R-TAS(D) sequence. Shown are eye colors of a $w^{1118} ; P\left\{w^{+}, r y^{+}\right\} A 4-4$ strain in wild-type, heterozygous, and homozygous piwi ${ }^{2}$ backgrounds. (Modified from Yin and Lin 2007 [@ Nature Publishing Group].)

A

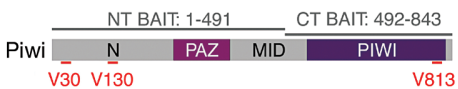

B

\begin{tabular}{|c|c|c|}
\hline PREY & \multicolumn{2}{|c|}{ PIWI BAIT } \\
\hline HP1a & WT & \\
\hline HP1a & V30A & 10.920 \\
\hline HP1a & V130A & (2) 8 \\
\hline HP1a & V30AN130A & 0.8 \\
\hline Control & WT & Q 0 \\
\hline
\end{tabular}

C

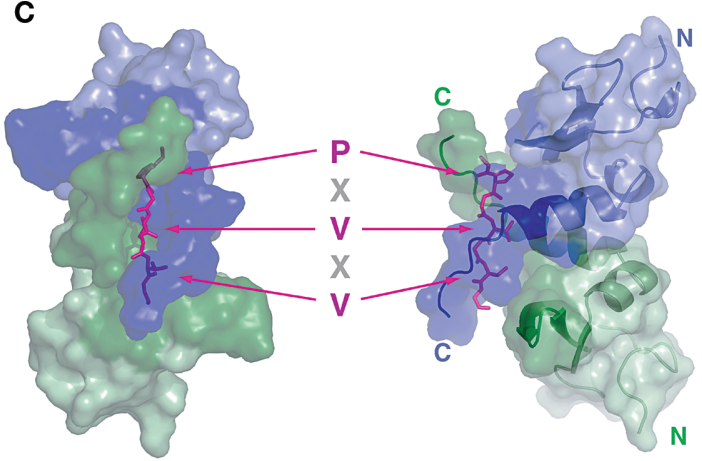

Figure 4. Piwi directly interacts with HP1a. (A) Baits used in PIWI Y2H screens. PIWI contains N, PAZ, MID, and PIWI domains. Y2H baits are PIWI-FL and residues 1-843, PIWI-NT and residues 1-491, and PIWI-CT and residues 492-843. The positions of the three "PXV" sequences, at V30, V130, and V813, are indicated. (B) V30 of PIWI but not V130 is required for HP1a interaction. Wildtype and V130A PIWI mutants produce comparably strong LacZ signals, whereas the signal in V30A, the V30A/V130A double mutant, or the negative control ("Control") is undetectable. (C) Model of the HP1a-chromoshadow domain (CSD) dimer in complex with the PIWI peptide (TSRGSGDGPRVKVFRGSSSGD). Top and side views are shown in left and right panels, respectively. Each monomer of the HP1a-CSD dimer is color-coded (green or blue). The CSD-binding motif (PXVXV) in the PIWI peptide is shown in stick models (magenta). Side chains of the conserved residues $(\mathrm{P}, \mathrm{V}, \mathrm{V})$ are displayed. Chemical shift perturbations are calculated as $\delta_{\mathrm{CS}}=\left(\delta_{\mathrm{H}}^{2}+\left(0.2 \delta_{\mathrm{N}}\right)^{2}\right)^{1 / 2}$. Residues of HP1a that experience significant resonance perturbation $\left(\delta_{\mathrm{CS}}>0.06 \mathrm{ppm}\right)$ during PIWI peptide titration are colored in dark green and dark blue and are mapped on the CSD surface and the ribbon diagram. The homology model of the complex was built using the XLOOK program and the figure was generated by PyMOL (Delano Scientific, South San Francisco, CA). (Modified from Brower-Toland et al. 2007.) 
Piwi-NT encoded HP1a, a key component of epigenetic silencing systems (James et al. 1989; Hiragami and Festenstein 2005).

HP1a binding to Piwi is strong and highly specific: HP1a does not interact with a panel of unrelated baits. HP1a also fails to interact with any of the four Piwi homologs in Drosophila (Aubergine, Ago1, Ago2, and Ago3; Brower-Toland et al. 2007). Conversely, Piwi interacts with HP1a but not with two other HP1a-like chromatin proteins, HP1b and HP1c.

Piwi-HP1a interaction domains were then mapped using a yeast two-hybrid assay and nuclear magnetic resonance (NMR) analysis. HP1a is known to bind to heterochromatin by interacting with the methylated $\mathrm{K} 9$ of histone 3 (H3K9me). This interaction is achieved by the amino-terminal chromodomain. In addition, HPla has a central hinge domain required for interaction with histone H1 and a carboxy-terminal chromoshadow domain (CSD) required for the dimerization of HP1a and interaction with many HP1 target proteins that contain a PXVXL motif (Smothers and Henikoff 2000; Lechner et al. 2005). Deletion analysis demonstrates that the HP1a CSD alone is necessary and sufficient for binding to NT- and FLPIWI baits.

This interaction was further confirmed using two Drosophila HP1a point mutations that should disrupt the interaction between the CSD dimmer and its target (Brower-Toland et al. 2007). In mice, it is known that two CSDs symmetrically dimerize to form the interface for the asymmetrical binding of a single target peptide (Thiru et al. 2004). The W200A mutant does not affect dimerization but abolishes HP1 binding to the PXVXL motif in targets, and the I191E mutant disrupts HP1 dimerization. PIWI binding is lost in both of these HP1 a mutants, suggesting that an intact CSD dimer interface is required for binding to PIWI. In contrast, PIWI interaction is maintained in the CD mutation V26M, which abolishes H3K9me binding. Thus, HP1a binding to methylated $\mathrm{H} 3 \mathrm{~K} 9$ is not required for PIWI binding.

Deletion analyses indicate that HP1a requires only the amino domain of PIWI for interaction. This domain contains two known vertebrate HP1-binding motifs: PRVKV centered on V30 and PRVRM centered on V130. Mutating V30 to alanine abolishes HP1a binding. In contrast, mutating V130 to alanine does not impact the interaction (Fig. 4B).

The direct interaction between the CSD of HP1a and the PRVKV motif centered on V30 was further confirmed by NMR titration of a 21-mer PIWI peptide centered on V30 with the HP1a CSD dimer (Brower-Toland et al. 2007). The exchange rate was slow on the NMR timescale, consistent with tight binding between the HP1aCSD dimmer and the PIWI peptide. These analyses allowed the construction of a structural model of a dimmer of the Drosophila HP1a CSDs, where a single PIWI peptide binds across the dimer of an HP1a CSD. CSD residues that experience significant resonance perturbation during NMR titration are primarily distributed along the $\beta$ strands close to the PIWI peptide, the carboxy-terminal portion of the central helices, and the carboxy-terminal extended loop. Together, these data strongly suggest that PIWI binds specifically to HP1a, which represents a direct interaction between an RNAi component and a protein of the canonical epigenetic machinery in any eukaryote.

\section{PIWI INTERACTS WITH HP1A AT MANY CHROMOSOMAL SITES IN SOMATIC CELLS}

Because both Piwi and HPla are chromatin-associated proteins in somatic cells, it is likely that their interaction occurs on chromosomes in these cells. To test this possibility and to assess the extent of Piwi-HP1a interaction on chromosomes, indirect immunofluorescence microscopy was conducted to examine Piwi-HPla colocalization on Drosophila salivary gland polytene chromosomes. This experiment revealed a complex banded distribution of PIWI on polytenes that is partially coincident with HP1a (Brower-Toland et al. 2007). Although HPla stained prominently localizes to the chromocenter (an agglomeration of pericentric heterochromatin) and to the largely heterochromatic fourth chromosome, HP1a is also found in all telomeres and in more than 200 bands along polytene chromosome arms. A prominent site of HP1a binding on chromosome arms is cytological region 31A, wherein HP1a localizes to eight discrete bands. PIWI colocalizes with HP1a in a complex pattern at multiple classes of chromatin loci, but it is completely absent from region 31A. Within the chromocenter, while HP1a is uniformly distributed, but PIWI has an irregular and particulate distribution within the pericentric heterochromatin of each chromosome. In chromosome 4, PIWI is restricted to a small number of distinct bands relative to HP1a, including one at or near the telomere. PIWI deposition also overlaps with HP1a in polytene telomeres. These data suggest that PIWI interacts with HP1 on chromosomes at many, but not all, genomic sites.

\section{PIWI-HP1A INTERACTION IS REQUIRED FOR THE EPIGENETIC EFFECT OF PIWI IN SOMATIC CELLS}

The direct interaction between Piwi and HP1a potentially represents a very direct means by which PIWI protein could act to recruit epigenetic effectors to specific sites in the Drosophila genome. To test the functional significance of PIWI-HP1a interaction in somatic cells, two assays were conducted (Brower-Toland et al. 2007). First, piwi transgenes encoding either the wild-type or V30A mutant form of piwi were introduced into a (lethal) piwinull genetic background. The wild-type transgene was able to rescue viability, whereas the V30A mutant transgene failed to rescue viability. Second, it has been shown that hypomorphic alleles of piwi act as dominant suppressors of heterochromatic gene silencing on chromosome 4 in somatic cells, as revealed by the expression of transgenic $w^{+}$reporter genes in the eye (Pal-Bhadra et al. 2004). This allowed the measurement of either transgene's ability to rescue dominant silencing defects produced in flies heterozygous for the protein-null piwi ${ }^{2}$ allele. In this haploinsufficient piwi background, the presence of the wild-type transgene supported greater silenc- 
ing of variegating white reporters than did the presence of the V30A transgene (see Fig. 6 below). These data strongly suggest that Piwi-HP1a interaction is directly involved in silencing white reporters embedded in constitutive heterochromatin in these somatic cells. In addition, they indicate that Piwi has opposing epigenetic effects toward different sites on the genome in somatic cells.

\section{PIWI IS REQUIRED FOR HP1A BINDING AND H3K9 METHYLATION AT MANY GENOMIC SITES IN SOMATIC CELLS}

We then investigated the relationship among PIWI, $\mathrm{HP} 1 \mathrm{a}$, and the epigenetic marker $\mathrm{H} 3 \mathrm{~K} 9 \mathrm{me}$. It has been long established that HP1a largely, but not exclusively, depends on the H3K9me marker provided by SUVAR3-9 family histone methyltransferases (HMTs) to bind to chromosomes. In addition, both HP1 targeting and H3K9me marking of many sites of polytene chromosomes have some dependence on piwi (Pal-Bhadra et al. 2004). This would suggest that Piwi acts upstream of H3K9 methylation and the binding of HP1a to these sites in these somatic cells. If so, we would expect H3K9me markers to colocalize with Piwi in polytene chromosomes, which indeed is the case (Brower-Toland et al. 2007). This, taken together with the fact that Piwi binds to HP1a and that Hp1a can bind to histone methylase, suggests the possibility that Piwi-piRNA recruits HP1a to these sites to initiate epigenetic marking. This then some- how further recruits $\mathrm{H} 3 \mathrm{~K} 9$ histone methylase, resulting in H3K9 methylation to further heterochromatinize PiwipiRNA target sites.

This mechanism would predict that PIWI localization to polytene chromosomes should not depend on the presence of HP1a and that PIWI binding to chromosomes is RNA dependent. Both predictions turn out to be true. In an HPla mutant where HP1a is not detectable, Piwi localization in chromatin is not globally altered (Fig. 5B). However, when polytene chromosomes are pretreated with RNase A, PIWI staining on chromatin is greatly diminished (Fig. 5C).

\section{PIWI/PIRNA-MEDIATED EPIGENETIC MECHANISM: THE PIWI-PIRNA GUIDANCE HYPOTHESIS}

The results reviewed above revealed a novel epigenetic pathway in somatic cells that is mediated by Piwi and its associated piRNAs and allowed us to propose the following "Piwi-piRNA guidance hypothesis" (Fig. 6): A PIWIpiRNA complex binds to piRNA-corresponding genomic sequences via either piRNA-DNA base pairing (by either a conventional or unconventional paring mechanism) or by a piRNA forming a duplex with a nascent noncoding RNA transcript being transcribed from the target genomic sequence. This leads to the direct recruitment of a HP1a molecule to the genomic site, which initiates heterochromatization. The PIWI-piRNA-HP1a complex then
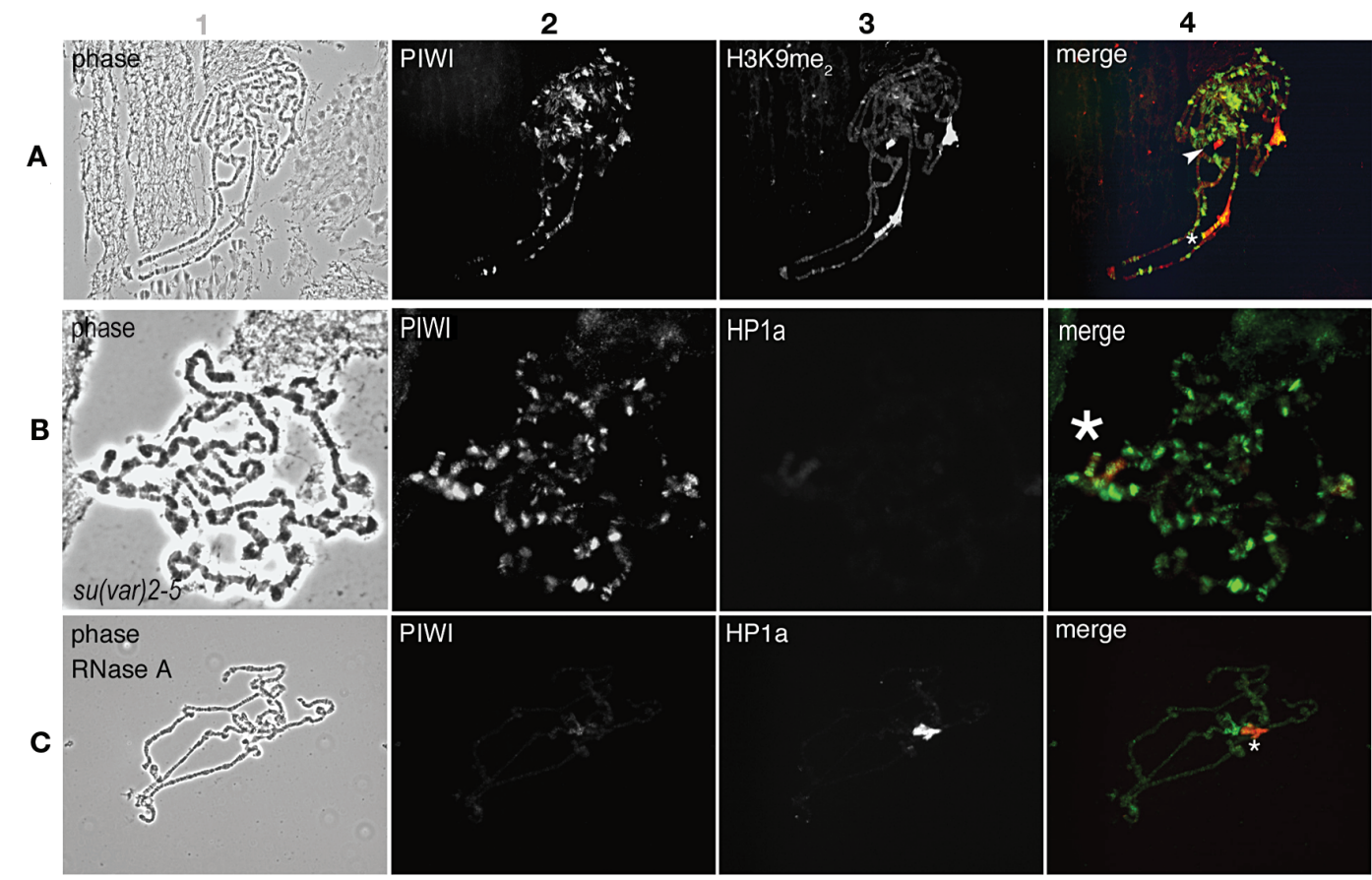

Figure 5. Chromatin binding of Piwi overlaps H3K9 methylation and depends on RNA hybrids but not HP1a. All images are of wildtype (Oregon R) chromosomes, except for $B$, which shows $S u(v a r) 2-5$ (the HP1a gene) mutant chromosomes. ( $A$ ) PIWI shows significant overlap with dimethyl-H3K9 in the chromocenter and fourth chromosome but not region 31 (white arrowhead). (B) PIWI localization to polytene chromosomes is not globally perturbed in the absence of HP1a. Because of their small size, Su(var)2-5 chromosomes are shown at higher magnification by comparison with wild-type chromosomes. (C) Mild treatment with RNase A eliminates the PIWI-binding pattern seen on untreated chromosomes without perturbing HP1a. (Adapted from Brower-Toland et al. 2007.) 


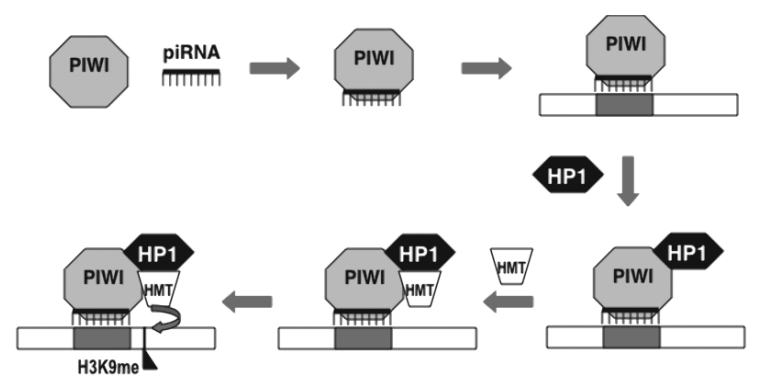

Figure 6. Novel epigenetic pathway mediated Piwi and piRNAs. For details, see text. Not shown are other factors possibly involved in the pathway.

further recruits HMTs such as SU(VAR)3-9 to methylate H3K9, which further leads to the stabilization and/or enhancement of heterochromatization.

This model is distinct from the RNAi model proposed in fission yeast (Volpe et al. 2002; Noma et al. 2004). Our model represents an $\mathrm{H} 3 \mathrm{~K} 9$ me-independent mode for initial HP1 localization, an alternative but potentially effective means of triggering formation of heterochromatin at specific genomic sites. In this model, the epigenetic activation effect of Piwi at certain sites in the genomes could be achieved by either recruiting an epigenetic activator or having HP1a exhibiting epigenetic activation function at particular genomic sites. Such a function has been previously reported (Hediger and Gasser 2006).

It remains possible that heterochromatin formation is guided by a different mechanism. In this case, the presence of HP1 a could allow stable binding of PIWI to heterochromatin for posttranscriptional gene silencing. It is also possible that the Piwi-piRNA-mediated mechanism acts at earlier developmental stages than the expressed epigenetic phenotype. In addition, there is evidence that Piwi proteins can also mediate other molecular mechanisms, especially when they are in the cytoplasm. Regardless of these possibilities, the Piwi-piRNA-mediated epigenetic mechanism, as revealed in somatic cells, may also be responsible for Piwi functions in the germ line, because Piwi and piRNAs are also localized in the nucleus in the germ line.

\section{CONCLUDING REMARKS}

Epigenetic programming of gene expression represents an exciting new frontier of stem cell research. A key yet essentially unexplored question in epigenetic programming is that how epigenetic regulators, most of which lack any DNA sequence recognition ability, are correctly guided to specific loci in the genome to exert their function. Recent studies suggest that transcriptional factors may have such a guidance role. Even so, this can only explain the targeting of a small fraction of the genome, because the vast majority of the genome is not gene coding. Our work in Drosophila somatic cells suggests that Piwi and its associated piRNAs guide epigenetic regulation to specific genomic sites. Indeed, this function of Piwi and its piRNAs may represent a major epigenetic guidance mechanism in Drosophila somatic cells, given the association of Piwi with many regions of polytene chromosomes and the sufficient complexity of Piwi-associated piRNAs in targeting diverse regions throughout the genome. However, this mechanism alone is probably insufficient to account for the epigenetic programming of the entire genome, because Piwi does not bind to all HP1a sites and HP1a may not participate in epigenetic programming at all genomic sites. Other questions also remain: Which regions of the Drosophila genome are regulated by the PiwipiRNA-mediated mechanism? How widely has the PiwipiRNA mechanism been adopted during the course evolution? Does this mechanism also exist in the germ line? Answers to these questions should have tremendous impact on stem cell and developmental biology.

\section{ACKNOWLEDGMENTS}

We are grateful to Seth Findley, Brent Brower-Toland, Sarah Elgin, Pei Zhou, and Liang Jiang whose work with us on the Piwi-HP1a interaction represents an important part of this review. We thank Jonathan Saxe, Travis Thomson, and Vamsi Gangaraju for critically reading the manuscript on short notice. The research in the Lin lab is supported by the National Institutes of Health (grants HD33760, HD33760-S1, and HD42012), the Connecticut Stem Cell Research Fund, and the Mathers Foundation Award.

\section{REFERENCES}

Aravin, A., Gaidatzis, D., Pfeffer, S., Lagos-Quintana, M., Landgraf, P., Iovino, N., Morris, P., Brownstein, M.J., KuramochiMiyagawa, S., Nakano, T., et al. 2006. A novel class of small RNAs bind to MILI protein in mouse testes. Nature 442: 203207.

Brennecke, J., Aravin, A.A., Stark, A., Dus, M., Kellis, M., Sachidanandam, R., and Hannon, G.J. 2007. Discrete small RNA-generating loci as master regulators of transposon activity in Drosophila. Cell 128: 1089-1103.

Brower-Toland, B., Findley, S.D., Jiang, L., Liu, L., Yin, H., Dus, M., Zhou, P., Elgin, S.C., and Lin, H. 2007. Drosophila PIWI associates with chromatin and interacts directly with HP1a. Genes Dev. 21: 2300-2311.

Cox, D.N. 1999. "Function of the Drosophila piwi gene in the selfrenewing division of germline stem cells and in germline development." Ph.D. thesis. Duke University, Durham, North Carolina.

Cox, D.N., Chao, A., and Lin, H. 2000. piwi encodes a nucleoplasmic factor whose activity modulates the number and division rate of germline stem cells. Development 127: 503-514.

Cox, D.N., Chao, A., Baker, J., Chang, L., Qiao, D., and Lin, H. 1998. A novel class of evolutionarily conserved genes defined by piwi are essential for stem cell self-renewal. Genes Dev. 12: 3715-3727.

Deng, W. and Lin, H. 2002. miwi, a murine homolog of piwi, encodes a cytoplasmic protein essential for spermatogenesis. Dev. Cell 2: 819-830.

Girard, A. and Hannon, G.J. 2008. Conserved themes in smallRNA-mediated transposon control. Trends Cell Biol. 18: 136148.

Girard, A., Sachidanandam, R., Hannon, G.J., and Carmell, M.A. 2006. A germline-specific class of small RNAs binds mammalian Piwi proteins. Nature 442: 199-202.

Grivna, S.T., Beyret, E., Wang, Z., and Lin, H. 2006. A novel class of small RNAs in mouse spermatogenic cells. Genes Dev. 20: 1709-1714.

Harris, A.N. and Macdonald, P.M. 2001. aubergine encodes a 
Drosophila polar granule component required for pole cell formation and related to eIF2C. Development 128: 2823-2832.

Hediger, F. and Gasser, S.M. 2006. Heterochromatin protein 1: Don't judge the book by its cover! Curr. Opin. Genet. Dev. 16: $143-150$.

Hiragami, K. and Festenstein, R. 2005. Heterochromatin protein 1: A pervasive controlling influence. Cell. Mol. Life Sci. 62: 2711-2726.

James, T.C., Eissenberg, J.C., Craig, C., Dietrich, V., Hobson, A., and Elgin, S.C. 1989. Distribution patterns of HP1, a heterochromatin-associated nonhistone chromosomal protein of Drosophila. Eur. J. Cell Biol. 50: 170-180.

Kim, V.N. 2006. Small RNAs just got bigger: Piwi-interacting RNAs (piRNAs) in mammalian testes. Genes Dev. 20: 19931997.

Kuramochi-Miyagawa, S., Kimura, T., Ijiri, T.W., Isobe, T., Asada, N., Fujita, Y., Ikawa, M., Iwai, N., Okabe, M., Deng, W., Lin, H., Matsuda, Y., and Nakano, T. 2004. Mili, a mammalian member of piwi family gene, is essential for spermatogenesis. Development 131: 839-849.

Lau, N.C., Seto, A.G., Kim, J., Kuramochi-Miyagawa, S., Nakano, T., Bartel, D.P., and Kingston, R.E. 2006. Characterization of the piRNA complex from rat testes. Science 313: 363 367.

Lechner, M.S., Schultz, D.C., Negorev, D., Maul, G.G., and Rauscher III, F.J. 2005. The mammalian heterochromatin protein 1 binds diverse nuclear proteins through a common motif that targets the chromoshadow domain. Biochem. Biophys. Res. Commun. 331: 929-937.

Liu, J., Carmell, M.A., Rivas, F.V., Marsden, C.G., Thomson, J.M., Song, J.J., Hammond, S.M., Joshua-Tor, L., and Hannon, G.J. 2004. Argonaute2 is the catalytic engine of mammalian RNAi. Science 305: 1437-1441

Liu, X., Sun, Y., Guo, J., Ma, H., Li, J., Dong, B., Jin, G., Zhang, J., Wu, J., Meng, L., and Shou, C. 2006. Expression of hiwi gene in human gastric cancer was associated with proliferation of cancer cells. Int. J. Cancer 118: 1922-1929.

Megosh, H.B., Cox, D.N., Campbell, C., and Lin, H. 2006. The role of PIWI and the miRNA machinery in Drosophila germline determination. Curr. Biol. 16: 1884-1894.

Motamedi, M.R., Verdel, A., Colmenares, S.U., Gerber, S.A., Gygi, S.P., and Moazed, D. 2004. Two RNAi complexes, RITS and RDRC, physically interact and localize to noncoding centromeric RNAs. Cell 119: 789-802.

Noma, K., Sugiyama, T., Cam, H., Verdel, A., Zofall, M., Jia, S., Moazed, D., and Grewal, S.I. 2004. RITS acts in cis to promote RNA interference-mediated transcriptional and post-transcriptional silencing. Nat. Genet. 36: 1174-1180.

Palakodeti, D., Smielewska, M., Lu, Y.C., Yeo, G.W., and Graveley, B.R. 2008. The PIWI proteins SMEDWI-2 and SMEDWI-3 are required for stem cell function and piRNA expression in planarians. RNA 14: 1174-1186.

Pal-Bhadra, M., Leibovitch, B.A., Gandhi, S.G., Rao, M., Bhadra, U., Birchler, J.A., and Elgin, S.C. 2004. Heterochromatic silencing and HP1 localization in Drosophila are dependent on the RNAi machinery. Science 303: 669-672.

Qiao, D., Zeeman, A.M., Deng, W., Looijenga, L.H., and Lin, H. 2002. Molecular characterization of hiwi, a human member of the piwi gene family whose overexpression is correlated to seminomas. Oncogene 21: 3988-3999.
Reddien, P.W., Oviedo, N.J., Jennings, J.R., Jenkin, J.C., and Sanchez Alvarado, A. 2005. SMEDWI-2 is a PIWI-like protein that regulates planarian stem cells. Science 310: 1327-1330.

Rossi, L., Salvetti, A., Lena, A., Batistoni, R., Deri, P., Pugliesi, C., Loreti, E., and Gremigni, V. 2006. DjPiwi-1, a member of the PAZ-Piwi gene family, defines a subpopulation of planarian stem cells. Dev. Genes Evol. 216: 335-346.

Saito, K., Nishida, K.M., Mori, T., Kawamura, Y., Miyoshi, K., Nagami, T., Siomi, H., and Siomi, M.C. 2006. Specific association of Piwi with rasiRNAs derived from retrotransposon and heterochromatic regions in the Drosophila genome. Genes Dev. 20: 2214-2222.

Sharma, A.K., Nelson, M.C., Brandt, J.E., Wessman, M., Mahmud, N., Weller, K.P., and Hoffman, R. 2001. Human CD34 stem cells express the hiwi gene, a human homologue of the Drosophila gene piwi. Blood 97: 426-434.

Smothers, J.F. and Henikoff, S. 2000. The HP1 chromo shadow domain binds a consensus peptide pentamer. Curr. Biol. 10: 27-30.

Sugiyama, T., Cam, H., Verdel, A., Moazed, D., and Grewal, S.I. 2005. RNA-dependent RNA polymerase is an essential component of a self-enforcing loop coupling heterochromatin assembly to siRNA production. Proc. Natl. Acad. Sci. 102: $152-157$.

Surani, M.A. 2001. Reprogramming of genome function through epigenetic inheritance. Nature 414: 122-128.

Thiru, A., Nietlispach, D., Mott, H.R., Okuwaki, M., Lyon, D., Nielsen, P.R., Hirshberg, M., Verreault, A., Murzina, N.V., and Laue, E.D. 2004. Structural basis of HP1/PXVXL motif peptide interactions and HP1 localisation to heterochromatin. EMBO J. 23: 489-499.

Unhavaithaya, Y., Hao, Y., Beyret, E., Yin, H., KuramochiMiyagawa, S., Nakano, T., and Lin, H. 2009. MILI, a piRNA binding protein, is required for germline stem cell self-renewal and appears to positively regulate translation. J. Biol. Chem. (in press).

Vagin, V.V., Sigova, A., Li, C., Seitz, H., Gvozdev, V., and Zamore, P.D. 2006. A distinct small RNA pathway silences selfish genetic elements in the germline. Science 313: 320-324.

Verdel, A., Jia, S., Gerber, S., Sugiyama, T., Gygi, S., Grewal, S.I., and Moazed, D. 2004. RNAi-mediated targeting of heterochromatin by the RITS complex. Science 303: 672-676.

Volpe, T.A., Kidner, C., Hall, I.M., Teng, G., Grewal, S.I., and Martienssen, R.A. 2002. Regulation of heterochromatic silencing and histone H3 lysine-9 methylation by RNAi. Science 297: 1833-1837.

Wang, Y., Juranek, S., Li, H., Sheng, G., Tuschl, T., and Patel, D.J. 2008. Structure of an argonaute silencing complex with a seed-containing guide DNA and target RNA duplex. Nature 456: $921-926$.

Wassenegger, M. 2005. The role of the RNAi machinery in heterochromatin formation. Cell 122: 13-16.

Watanabe, T., Takeda, A., Tsukiyama, T., Mise, K., Okuno, T., Sasaki, H., Minami, N., and Imai, H. 2006. Identification and characterization of two novel classes of small RNAs in the mouse germline: Retrotransposon-derived siRNAs in oocytes and germline small RNAs in testes. Genes Dev. 20: 1732-1743.

Yin, H. and Lin, H. 2007. An epigenetic activation role of Piwi and a Piwi-associated piRNA in Drosophila melanogaster. Nature 450: 304-308. 


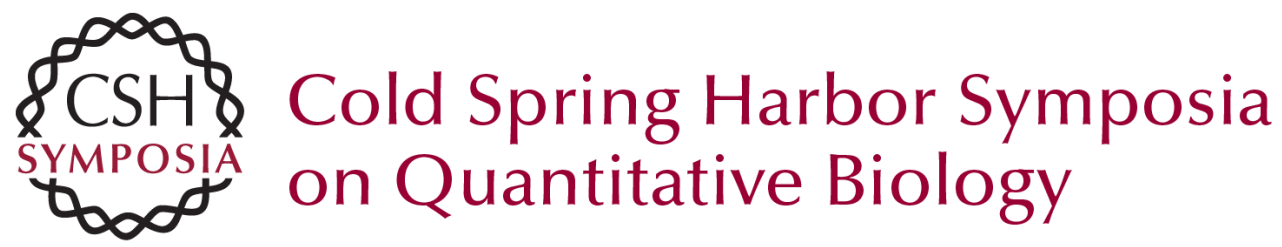

\section{A Novel Epigenetic Mechanism in Drosophila Somatic Cells Mediated by PIWI and piRNAs}

$\mathrm{H}$. Lin and $\mathrm{H}$. Yin

Cold Spring Harb Symp Quant Biol 2008 73: 273-281 originally published online March 6, 2009 Access the most recent version at doi:10.1101/sqb.2008.73.056

References This article cites 40 articles, 20 of which can be accessed free at: http://symposium.cshlp.org/content/73/273.full.html\#ref-list-1

\section{License}

Email Alerting Receive free email alerts when new articles cite this article - sign up in the box at the Service top right corner of the article or click here. 